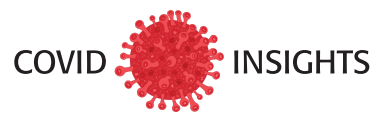

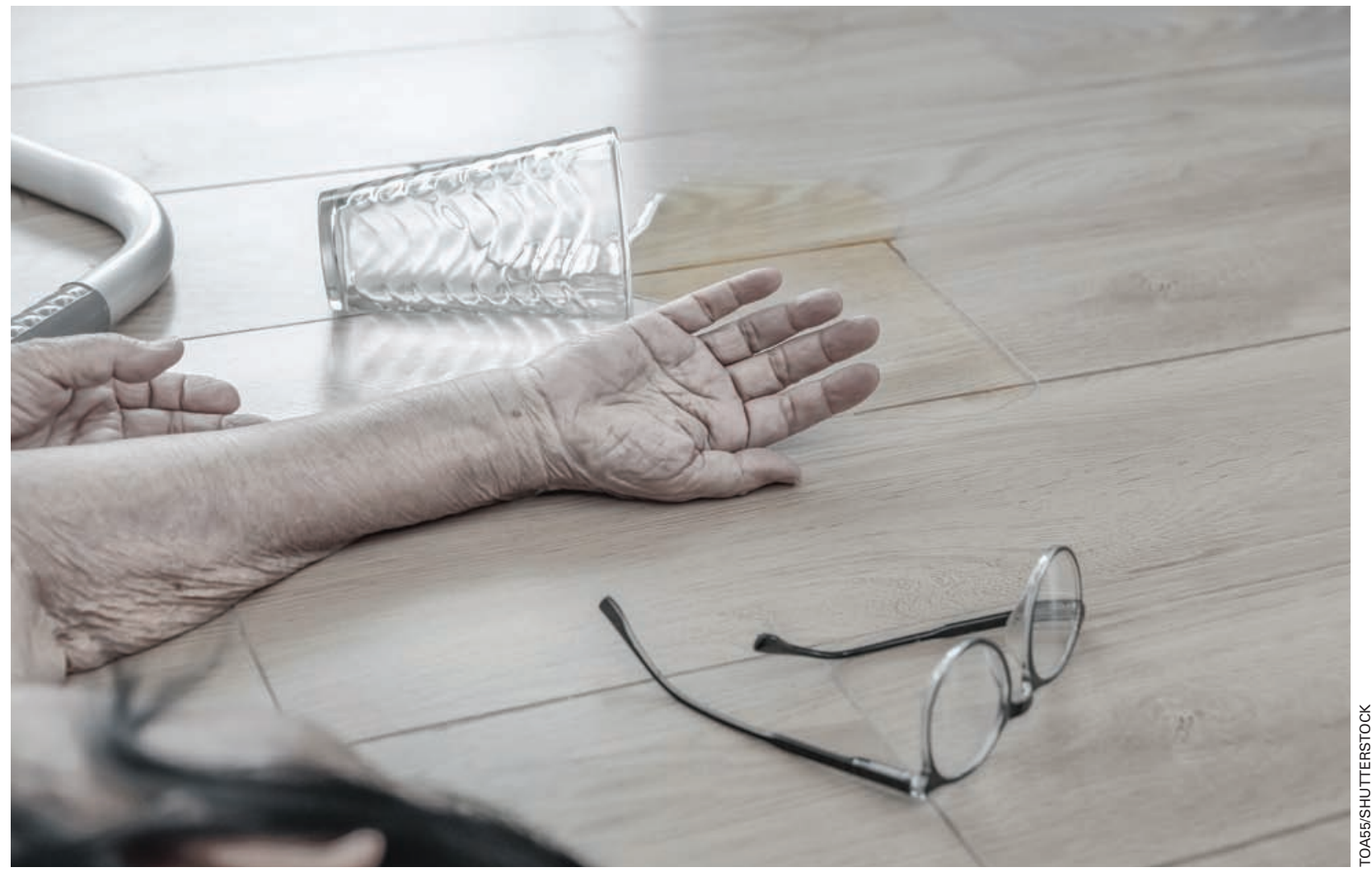

\title{
The impact of COVID-19 on patient
}

\section{safety attendant use}

BY LISA B.E. SHIELDS, MD; TIFFANY P. LAWSON, BSN, RN, CHPN; AND KIMBERLY FLANDERS, DNP, MBA, RN, NEA-BC

Abstract: Patient safety attendants (PSAs) provide constant direct observation to patients who have cognitive impairments or thoughts. Some estimates report that an acute care hospital in the United States may spend more than $\$ 1$ million annually on PSAs, an expenditure often not reimbursed. With no national defined standards to regulate or monitor PSA use, this study sought to determine the impact of COVID-19 on a PSA reduction program in a large Midwestern healthcare system.

Keywords: COVID-19, fall prevention, patient safety attendants, SARS-CoV-2, video monitoring

\section{SINCE THE EMERGENCE of the} first four cases of "pneumonia of unknown etiology" in Wuhan, China, on December 29, 2019-which were identified as the novel coronavirus (2019-nCoV) or SARS-CoV-2 - this pandemic has increased exponentially worldwide. ${ }^{1-5}$ As of August 1, 2021 , COVID-19 had affected 220 countries, with a total of 198,022,041 confirmed cases and 4,223,460 deaths. ${ }^{6}$ The Commonwealth of Kentucky reported 483,719 positive cases of COVID-19 with 7,378 virus-related deaths as of August 1, $2021 .^{7}$
In the American Nurses Association's survey of 32,000 nurses in March-April 2020, 87\% feared going to work, $36 \%$ cared for a patient diagnosed with COVID-19 without adequate personal protective equipment, and only $11 \%$ believed they were well prepared to care for patients with COVID-19. ${ }^{8}$ Because nurses are often frontline healthcare workers, they are at a heightened risk of infection, which jeopardizes their lives and those of their loved ones. The American Psychiatric Nurses Association has noted that nurses have ex- 
perienced pressure, fear, exhaustion, isolation, and emotional trauma during the COVID-19 pandemic. ${ }^{9}$ The physical and mental fatigue, coupled with a lack of sufficient education to treat patients with COVID-19, has prompted some nurses to refuse to work or to leave the profession. 8,10

We have previously reported how our healthcare system in Kentucky managed patients with COVID-19. ${ }^{11}$ Well-intentioned programs that were implemented by nurses prior to the COVID-19 pandemic were adversely affected by new institutional guidelines. In response to COVID-19, our healthcare system implemented a no-visitor policy on March 21, 2020, with few exceptions. All exempted hospital visitors were screened for signs and symptoms of COVID-19 and denied access if the screening was positive. These visitors were then referred to their primary care provider for follow-up assessment and care.

There are no national defined standards to regulate or monitor patient safety attendant (PSA) use. The current study sought to determine the impact of COVID-19 on the PSA reduction program at our institution. We also sought to analyze the use of video monitoring and other alternatives to PSA.

\section{Background}

PSAs, also known as sitters or observation assistants, provide constant direct observation of patients who have cognitive impairments or thoughts of harming themselves or others. ${ }^{12-16}$ These patients may experience hallucinations, psychosis, agitation, confusion, impulsiveness, restlessness, disorientation, or delirium, or they may be inclined to remove medical devices such as I.V. lines and catheters. ${ }^{12,16,17}$ PSAs are often unlicensed assistive healthcare providers who manage patients previously controlled by being placed in restraints. ${ }^{17,18}$ It has been

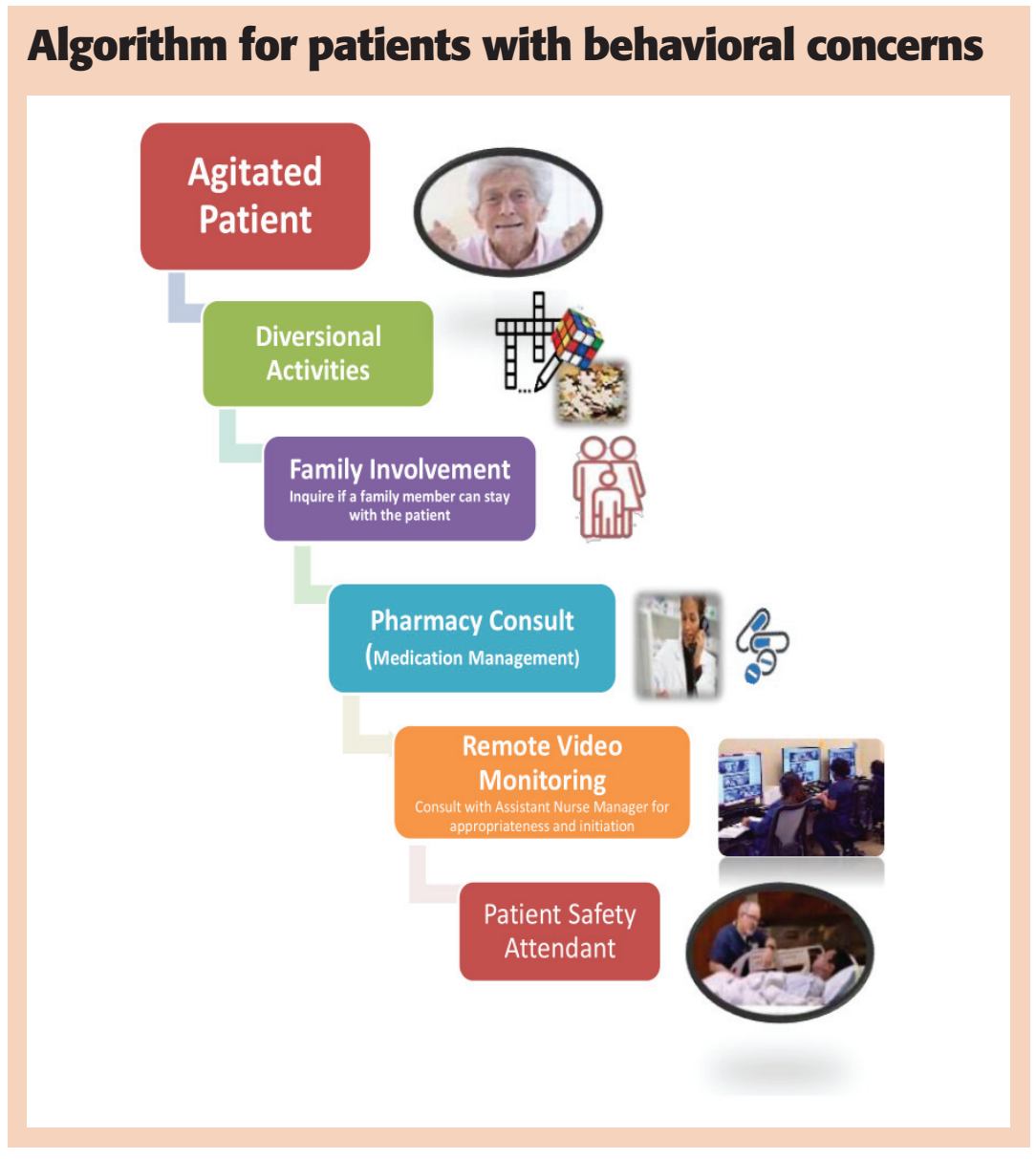

reported that an acute care hospital in the US may spend more than \$1 million annually on PSAs, an expenditure that often is not reimbursed by thirdparty payers. ${ }^{17-20}$

Falls represent one of the most common causes of morbidity, affecting 13\%-23\% of hospitalized patientsprimarily older adults with cognitive impairment. ${ }^{21}$ In 2015, the estimated medical costs attributable to fatal and nonfatal falls were approximately $\$ 50$ billion. ${ }^{22}$ The impact of PSAs on patient fall rates varies. Although a decline in falls has been attributed to PSAs, most studies have shown that a reduction in PSAs does not increase or decrease fall rates. ${ }^{15,17,20,21,23,24}$ Some studies have shown that PSAs have not received adequate fall prevention training. ${ }^{14,19,21}$ The PSA often does not per- form interventions to address the root cause of the problem, such as delirium. Video monitoring, specifically the use of portable cameras with a speaker and a trained technician to directly observe patients from a remote location, serves as an alternative to the PSA. ${ }^{25}$ Video monitoring has been shown to reduce PSA use and cost, although its impact on the number of patient falls varies in the literature. ${ }^{19,25-28}$

\section{Methods}

Under an Institutional Review Boardapproved protocol, our prospective study (January 1, 2017-October 31, 2020) analyzed PSA use at our institution. We focused on PSA hours for patients who exhibited confusion, agitation, and restlessness. PSA hours for patients monitored for suicidal 


\section{Number of patient safety attendant hours in a single neuro spine unit (November 2017-October 2020)}

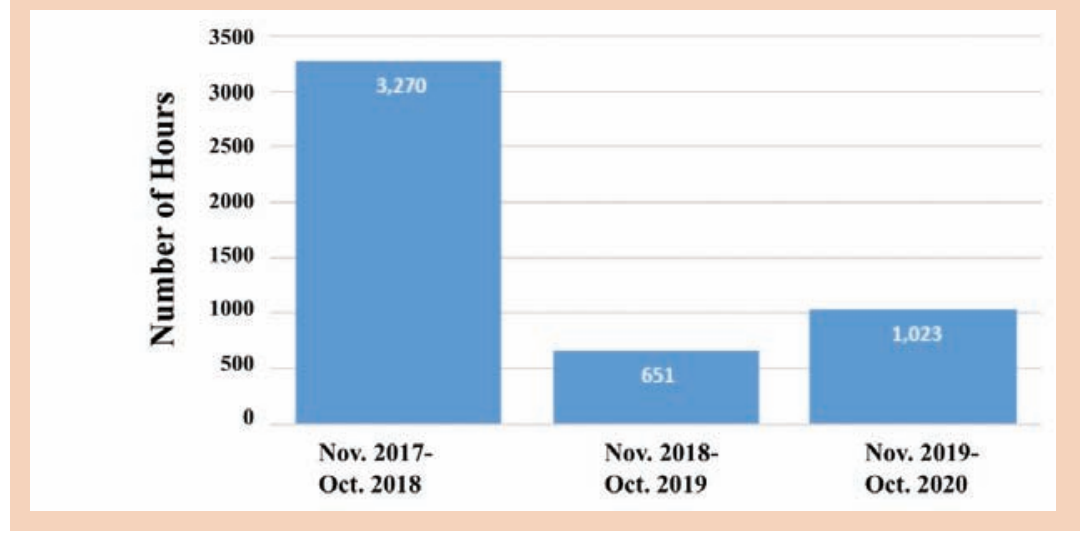

\section{Number of patient safety hours in 10 medical units (April 2018 - March 2021 [Annualized])}

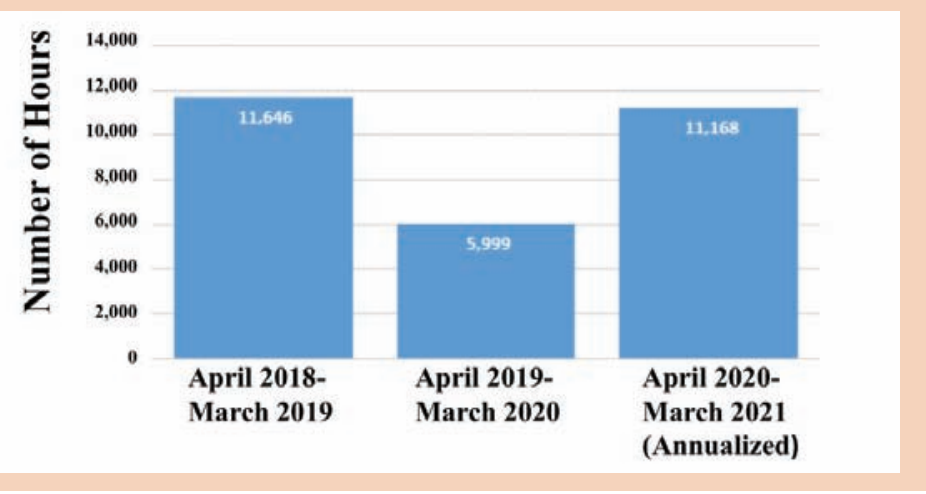

\section{Diversional activity} box

- Activity pillowcase

- Baby doll

- Coloring pages/crossword puzzles

- Playing cards

- Stress relief heart

- Lavender plug-in

- Shapes cube

- Toolkit

- Rubik's Cube

- Magazines/soft books

- Bubble watcher

- Twisting tubes

- Baby activity cube

- Slinky

- White noise/music ideation and during a 72-hour hold were excluded. Patient-care associates (PCAs) were the designated PSAs. Prior to the initiation of this study, our institution observed a remarkably high number of PSA hours associated with a substantial cost. In May 2018, a Sitter Project Group was developed with the goals of (1) curtailing the number of PSA hours while ensuring patient safety, (2) placing PCAs back on the medical units to provide patient care, (3) creating a cost savings, and (4) enhancing communication and cooperation between nurses, PCAs, PSAs, patients, and patients' families.
The PSA reduction program was a nurse-driven intervention. The Sitter Project Group consisted of two assistant nurse managers (ANMs), two PCAs, a nurse educator, two unit nurses, and a process improvement coach. An algorithm was developed for the nursing staff to follow, and a diversional activity box was created. (See Algorithm for patients with behavioral concerns and Diversional activity box.)

When a patient exhibited concerning behavior, the first step was to contact the ANM and thoroughly assess the situation to ascertain the reason for the agitation or restlessness. Determining whether a patient had a physical problem or was climbing out of bed because of a full bladder was an important component to address when evaluating a patient's behavioral issues. A pharmacy consult was also obtained to confirm appropriate medication management.

All inpatient unit managers and ANMs at our institution were educated about the process and use of interventions. All inpatient areas adopted the same mentoring with staff to encourage them to use alternative activities instead of a PSA. The PSA reduction program, implemented in our Neuro Spine unit in November 2018, was expanded to 10 medical inpatient units (cardiac, acute specialty care, medical-surgery, and oncology) in April 2019. The video monitoring program was incorporated in December 2018. Three telemonitors are used to monitor patients and are not a substitute for people. The camera option alerts staff when a patient is moving, so that staff can reach the patient sooner and intervene. A video monitoring algorithm was established to ensure that the three telemonitors are in place and utilized for the highestrisk patients. Our institution does not provide the actual cost savings of PSA reduction, so we presented the cost savings as a percentage change. We 
also reported the number of falls sustained by patients at our institution.

\section{Results}

PSA hours in a single neuro spine unit at our institution. The number of PSA hours in a single Neuro Spine unit substantially decreased during the first year after the PSA reduction program was implemented, from 3,270 hours (November 2017-October 2018, prior to the start of the PSA reduction program) to 651 hours (November 2018-October 2019). (See Number of patient safety attendant hours in a single neuro spine unit [November 2017-October 2020].) The sitter hours from November 2019 to October 2020 increased to 1,023 hours, reflecting a $68 \%$ reduction compared with the baseline year.

PSA hours in 10 medical units at our institution. The number of PSA hours in all medical units at our institution greatly declined during the first year after the PSA reduction program was implemented in all 10 medical units, from 11,646 hours (April 2018-March 2019, prior to introduction of the PSA reduction program in all 10 medical units) to 5,999 hours (April 2019-March 2020). (See Number of patient safety attendant hours in 10 medical units, April 2018-March 2021 [Annualized].)

The annualized sitter hours to March 2021 (using real data from April through October 2020) increased to 11,168 hours, representing a $4 \%$ decrease in the number of sitter hours compared with the baseline year. The first year of our study sustained significant gains. With visitor restrictions and practice changes related to COVID-19, our PSA hours increased. They did not return to our initial preintervention.

Cost savings for PSAs at our institution. During the first year of the PSA reduction program in the single Neuro Spine unit, PSA expenditure

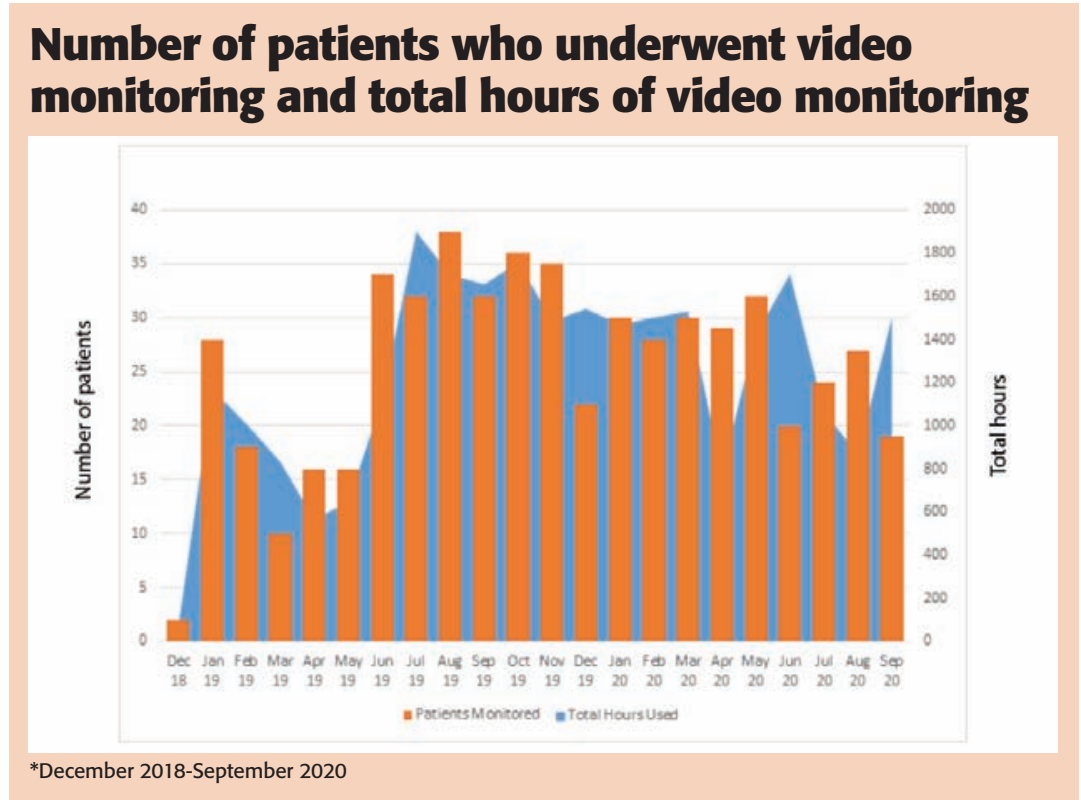

\section{Utilization percentages and STAT alarm responses}

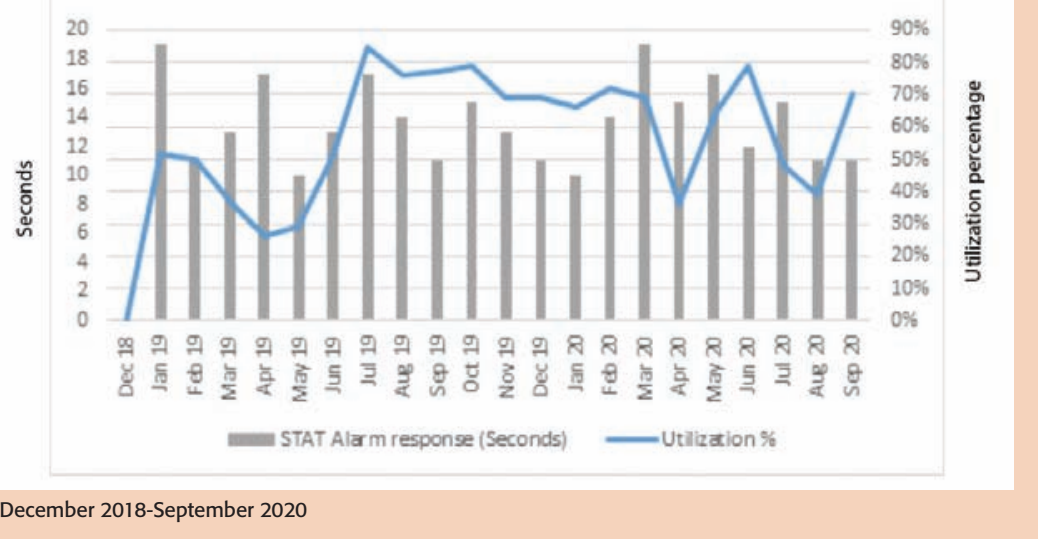

decreased by $80 \%$. During the second year, PSA expenditure for the single Neuro Spine unit was 68\% lower than the baseline year. For all 10 medical units at our institution, the PSA expenditure decreased by $48 \%$ during the first year of the PSA reduction initiative. The annualized second-year PSA expenditure for all 10 units is projected to be a $4 \%$ reduction from the baseline year.
Video monitoring at our institution. The number of patients who underwent video monitoring, total hours of video monitoring, utilization percentage (percentage of time that the video monitors were in use), and STAT alarm responses (the time it takes the monitor tech to respond to a STAT alarm) remained consistent throughout this study. STAT alarms required having an individual physi- 


\begin{tabular}{l} 
Number of Inpatient falls, excluding \\
\begin{tabular}{lllll}
\hline Metrics & $\mathbf{2 0 1 7}$ & $\mathbf{2 0 1 8}$ & $\mathbf{2 0 1 9}$ & $\mathbf{2 0 2 0}$ \\
\hline Total falls & 280 & 259 & 277 & 216 \\
\hline Total falls with injury & 41 & 49 & 50 & 48 \\
\hline Total falls unassisted & 240 & 222 & 236 & 182 \\
\hline Patient days & 90,448 & 86,819 & 86,173 & 60,667 \\
\hline Total falls/1000 patient days & 3.1 & 2.98 & 3.21 & 3.56 \\
\hline Total falls with injury/1000 patient day & 0.45 & 0.56 & 0.58 & 0.79 \\
\hline Total falls unassisted/1000 patient days & 2.65 & 2.56 & 2.74 & 3.00 \\
\hline *Through September 30, 2020 & & & &
\end{tabular} \\
\hline
\end{tabular}

cally on the medical unit to intervene. (See Number of patients who underwent video monitoring and total hours of video monitoring and Utilization percentages and STAT alarm responses.

Inpatient falls at our institution. The number of inpatient falls at our institution, excluding psychiatric units, remained consistent during the course of this study. (See Number of inpatient falls, excluding psychiatric units, 2017-2020.)

\section{Discussion}

Several studies have described PSA reduction strategies at different hospitals, but none has reported implementing a PSA reduction program while facing the challenges inherent in a worldwide pandemic. ${ }^{12,20,29}$ Adams and colleagues reported that the PSA reduction program at their single hospital resulted in \$1.2 million in annual savings without negatively impacting fall rates and restraint use. ${ }^{12}$ They attributed their program's success to the intentional presence of nursing staff, including altering the nurses' perceptions that PSAs were not needed to prevent patient falls. Following Spiva and colleagues' application of a PSA reduction program, they observed a significant decrease in PSA hours and costs without increasing fall rates. ${ }^{20}$ They developed an algorithm to assess which high-risk patients would most benefit from a PSA based on physiologic, psychosocial, and pharmacologic reasons for the patients' behavior. In this respect, nurses ascertained through an individualized approach which patients needed a PSA, thereby reducing the number of PSAs and the associated costs. By contrast, Tzeng and colleagues reported an increase in PSA requests and less use of restraints but a higher total fall rate following adoption of their Patient Attendant Assessment Tool (PAAT) ${ }^{29}$ The PAAT was developed to determine which patients need constant observation by a PSA. These authors concluded that PSAs were not cost-effective for decreasing patient falls because the gains did not offset the direct expense of their sitter program. They attributed the greater number of falls to the PSAs' lack of professional nursing education and stated that PSAs cannot replace nurses in preventing inpatient falls.

In an effort to curtail the use of PSAs, several alternatives have been reported in the literature, including relocating the patient's room closer to the nurses' station, installing personal bed and chair alarms, creating diversional activities, using relaxation techniques, wrapping I.V. lines, and installing bed enclosure devices. ${ }^{12,19,20,24}$ A patient's family members play a pivotal role in closely monitoring and providing emotional support. However, families do not have the professional training to avert falls. A close relationship and enhanced communication between nursing staff and family are imperative to ensure that relatives remain at the patient's bedside as a safety precaution and to inform nurses of any alterations in the patient's mental or physical status.

A mobile video monitoring program has been shown to be a successful alternative to PSAs. ${ }^{25,26,28}$ Burston and colleagues reported that implementation of a nurse-driven PSA protocol and video monitoring program led to a $47.1 \%$ reduction in combined sitter and video monitor technician costs. ${ }^{26}$ The video monitoring provided an additional safety benefit for the nursing staff and highrisk patients. Similarly, Cournan and colleagues reported that video monitoring improved patient safety by decreasing falls; reducing PSA usage and cost; and enhancing patient, family, and staff satisfaction. ${ }^{25}$ Votruba and colleagues noted that remote video monitoring was more effective than patient companions alone in reducing falls because more patients could be directly observed at all times. ${ }^{28}$ There was a decrease in $1: 1$ PSA use and a 35\% decline in falls. In the present study, the use of video monitoring remained consistent. 
Prior to the COVID-19 pandemic, Rochefort and colleagues reported that higher rates of RN overtime and lower RN cumulative inexperience were associated with greater PSA use. ${ }^{17}$ Each additional hour of RN overtime increased the likelihood of PSA use by $108 \%$, and the odds of PSA use were reduced by $23 \%$ for every 5 years of collective RN experience. Additionally, lower levels of worked hours per patient per day by RNs and PCAs were each associated with an increased likelihood of high PSA use costs. ${ }^{18}$ RNs' vigilance declined with working long hours, which increased their susceptibility to errors.

In the era of COVID-19, nurses' responsibilities have been potentiated by the extraordinary volume of patients who have sought medical care for life-threatening illnesses. Nurses' tension and anxiety have been exacerbated by the demands of learning new practices necessitated by COVID-19. Our institution's no-visitor policy due to COVID-19 led to a lack of family presence by the patients' bedside. $\mathrm{Pa}$ tients were isolated and did not have the safety measures and emotional encouragement offered by their families. Furthermore, the familiar voices and routines associated with families help patients recover.

\section{Strengths and limitations of the study}

The strength of the current study was the success of our nurse-driven PSA reduction program in the first year following its implementation, as reflected by the decreased number of PSAs, steep reduction in costs, and stable fall rate. Our study serves as a model that may be applied to other hospitals. Our findings indicate that family members of hospitalized patients play an important role as care partners. Because $\mathrm{CO}$ VID-19 prevented families from being present in the hospital, more PSAs were needed. Our findings also indi-

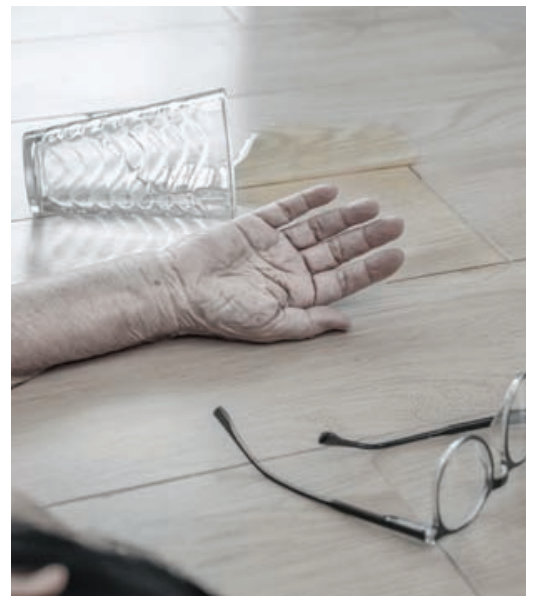

Falls represent one of the most common causes of morbidity, affecting $13 \%-23 \%$ of hospitalized patients-primarily older adults with cognitive impairment.

cate that the primary intervention is identifying the root cause of the need for a PSA. A physician intervention or medication adjustment may often correct the cause.

The primary limiting factor of our study was beyond our control. Our program lost ground because of the repercussions triggered by COVID-19. PSA use increased in the second year after initiation of the PSA reduction program because family members were not permitted to enter patients' rooms. With the rise in PSAs due to COVID-19, there was also a simultaneous cost increase. The projected PSA expenditure between April 2020 and March 2021 was based on the data from April 2020 to July 2020, when family members were prevented from assuming the PSA role. We anticipate that the actual PSA expenditure will be less than the projected amount because more liberal visitation occurred in late June 2020. Additional limitations consisted of nursing staff who were initially resistant to change because of the perceived increased workload with fewer PSAs and physicians who were accustomed to ordering PSAs instead of using a nurse-driven program.

\section{Conclusion}

A multifaceted approach is warranted when deciding whether a patient with behavioral concerns needs a PSA, followed by frequently reassessing PSA use. Selecting alternatives to PSAs such as video monitoring or a diversional activity box should also be considered prior to assigning a PSA. Additionally, adequate training for PSAs is recommended so that they may play a more active role in preventing falls. Despite multidisciplinary efforts to decrease PSA use, the challenging and evolving environment in the setting of COVID-19 impeded our abilities. While patients' family members represent valuable sources of monitoring and lessen the PSA demand, their assistance was not permitted during COVID-19 because preventing the spread of this potentially fatal disease was of paramount importance. Although nurses have adapted to the constantly evolving environment of COVID-19, the same primary goals of ensuring the safety and security of patients and supporting the emotional well-being of nurses are of utmost importance. Further research into factors that decrease the use of PSA is necessary.

REFERENCES

1. Cheng ZJ, Shan J. 2019 novel coronavirus: where we are and what we know. Infection. 2020;48(2):155-163.

2. Holshue ML, DeBolt C, Lindquist S, et al. First case of 2019 novel coronavirus in the United States. N Engl J Med. 2020;382(10):929-936.

3. Li Q, Guan X, Wu P, et al. Early transmission dynamics in Wuhan, China, of novel coronavirusinfected pneumonia. N Engl J Med. 2020;382: 1199-1207.

4. Singhal T. A review of coronavirus disease-2019 (COVID-19). Indian J Pediatr. 2020;87(4):281-286. 
5. Wiersinga WJ, Rhodes A, Cheng AC, Peacock SJ, Prescott HC. Pathophysiology, transmission, diagnosis, and treatment of coronavirus disease 2019 (COVID-19): a review. JAMA. 2020;324(8):782-793

6. World Health Organization. Coronavirus disease (COVID-19) pandemic. www.who.int/emergencies/ diseases/novel-coronavirus-2019.

7. Kanik A. Looking for county-level data on the coronavirus? Here's our Kentucky COVID-19 tracker https://wfpl.org/looking-for-county-level-data-on-the coronavirus-heres-our-kentucky-covid-19-tracker/

8. American Nurses Association. Survey: nurses fear going to work due to lack of protection from virus more than $32 \mathrm{k}$ nurses share experience from the front lines. www.nursingworld.org/news/news releases/2020/survey-nurses-fear-going-to-workdue-to-lack-of-protection-from virus-more-than-

32k-nurses-share-experience-from-the-front-lines/.

9. American Psychiatric Nurses Association

Managing stress \& self-care during COVID-19:

information for nurses. www.apna.org $/ \mathrm{m} /$ pages. cfm?pageID $=6685$

10. Schutz V, Shattell M. Impact of COVID-19: what does it mean for nurses and health systems? J Psychosoc Nurs Ment Health Serv. 2020;58(8):2-3.

11. Shields LBE, Hester ST, Schulz PS, et al. Healthcare system approach to managing COVID-19 in a metropolitan community in Kentucky. Medicine (Baltimore). 2020:99(38):e22254

12. Adams J, Kaplow R. A sitter-reduction program in an acute health care system. Nurs Econ. 2013;31(2):83-89.

13. Carr FM. The role of sitters in delirium: an update. Can Geriatr J. 2013;16(1):22-36.
14. de Jong LD, Weselman T, Kitchen S, Hill A-M Exploring hospital patient sitters' fall prevention task readiness: a cross-sectional survey. J Eval Clin Pract. 2020;26(1):42-49.

15. Harding AD. Observation assistants: sitter effectiveness and industry measures. Nurs Econ. 2010;28(5):330-336

16. Laws D, Crawford CL. Alternative strategies to constant patient observation and sitters: a proactive approach. J Nurs Adm. 2013;43(10):497-501

17. Rochefort CM, Ward L, Ritchie JA, Girard N, Tamblyn RM. Registered nurses' job demands in relation to sitter use: nested case-control study. Nurs Res. 2011;60(4):221-230.

18. Rochefort CM, Ward L, Ritchie JA, Girard N, Tamblyn RM. Patient and nurse staffing characteristics associated with high sitter use costs. J Adv Nurs. 2012;68(8):1758-1767.

19. Greeley AM, Tanner EP, Mak S, Begashaw MM, Miake-Lye IM, Shekelle PG. Sitters as a patient safety strategy to reduce hospital falls: a systematic review. Ann Intern Med. 2020;172(5):317-324.

20. Spiva L, Feiner T, Jones D, Hunter D Petefish J, VanBrackle L. An evaluation of a sitter reduction program intervention. J Nurs Care Qual. 2012;27(4):341-345.

21. de Jong LD, Kitchen S, Foo Z, Hill A-M. Exploring falls prevention capabilities, barriers and training needs among patient sitters in a hospital setting: pilot survey. Geriatr Nurs. 2018;39(3):263-270.

22. Florence CS, Bergen G, Atherly A, Burns E, Stevens J, Drake C. Medical costs of fatal and nonfatal falls in older adults. J Am Geriatr Soc. 2018:66(4):693-698.
23. Boswell DJ, Ramsey J, Smith MA, Wagers B. The cost-effectiveness of a patient-sitter program in an acute care hospital: a test of the impact of sitters on the incidence of falls and patient satisfaction. Qual Manag Health Care. 2001;10(1):10-16.

24. Lang CE. Do sitters prevent falls? A review of the literature. J Gerontol Nurs. 2014;40(5):24-33.

25. Cournan M, Fusco-Gessick B, Wright L. Improving patient safety through video monitoring. Rehabil Nurs. 2018;43(2):111-115.

26. Burtson PL, Vento L. Sitter reduction through mobile video monitoring: a nurse-driven sitter protocol and administrative oversight. J Nurs Adm. 2015:45(7-8):363-369.

27. Jeffers S, Searcey P, Boyle K, et al. Centralized video monitoring for patient safety: a Denver Health Lean journey. Nurs Econ. 2013;31(6):298-306.

28. Votruba L, Graham B, Wisinski J, Syed A. Video monitoring to reduce falls and patient companion costs for adult inpatients. Nurs Econ. 2016;34(4):185-189.

29. Tzeng H-M, Yin C-Y, Grunawalt J. Effective assessment of use of sitters by nurses in inpatient care settings. J Adv Nurs. 2008;64(2):176-183.

Lisa B.E. Shields is a medical research associate at Norton Neuroscience Institute, Louisville, Ky. Tiffany Lawson is a nurse manager for neuro spine at Norton Hospital, Louisville, Ky. Dr. Kimberly Flanders is the vice president of patient care services at Norton vospital, Louisville, Ky.

The authors have disclosed no financial relationships related to this article.

DOI-10.1097/01 NURSE.0000791760.49330.35 